\title{
DÜBLIN
}

Technological University Dublin ARROW@TU Dublin

1998-01-01

\section{White Light Interferometric Surface Profiler}

Vincent Toal

Technological University Dublin, vincent.toal@tudublin.ie

Brian Bowe

Technological University of Dublin, Brian.Bowe@TUDublin.ie

Follow this and additional works at: https://arrow.tudublin.ie/cieoart

Part of the Semiconductor and Optical Materials Commons

\section{Recommended Citation}

Bowe, B., Toal, V. (1998) White light interferometric surface profiler. Optical Engineering, Vol. 37, pp.1796. doi:10.1117/1.601727

This Article is brought to you for free and open access by the Centre for Industrial and Engineering Optics at ARROW@TU Dublin. It has been accepted for inclusion in Articles by an authorized administrator of ARROW@TU Dublin. For more information, please contact arrow.admin@tudublin.ie, aisling.coyne@tudublin.ie, gerard.connolly@tudublin.ie.

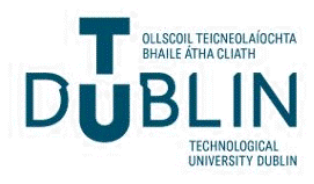




\section{White light interferometric surface profiler}

\author{
Brian Bowe \\ Vincent Toal \\ Dublin Institute of Technology \\ Kevin Street \\ Dublin 8 \\ Ireland \\ E-mail: bbowe@dit.ie
}

\begin{abstract}
We describe an optical system for 3-D profilometry based on the white light interferometer. Recently many different methods have been used to analyze the data obtained from white light interferometric profilers. Many commercially available white light profilers are also in use today. We detail a simple way to construct a profiler that uses two simple and efficient algorithms. It deals with the data in a fast and simple manner, thus reducing both the acquisition and analysis time. The system has a theoretically unlimited range and can profile both optically rough and smooth surfaces. (c) 1998 Society of Photo-Optical Instrumentation Engineers. [S0091-3286(98)02006-6]
\end{abstract}

Subject terms: white light interferometry; coherence region; profilometry.

Paper 07107 received Oct. 4, 1997; revised manuscript received Jan. 21, 1998; accepted for publication Jan. 21, 1998. Portions of this work were presented at the 1996 Applied Optics and Optoelectronics conference in Reading, England.

\section{Introduction}

The benefits of optical profilometry, compared to mechanical profilometry, include the ability to perform noncontact measurements of delicate surfaces, increased height resolution, and high-speed measurement. Optical interferometric surface profilers are widely used for 3-D surface profiling. These profilers usually involve phase-shifting interferometric (PSI) techniques ${ }^{1-4}$ to obtain 3-D images of surfaces that are smooth relative to the mean wavelength of the monochromatic source. A number of techniques have been developed to extend this range including heterodyne interferometry and multiple interferometry. ${ }^{5-10}$ Many papers detail a wide variety of algorithms that can be used with these profilers. ${ }^{3,4,11,12}$ However, these methods are still limited to ranges of the order of $10 \mu \mathrm{m}$.

A number of techniques that use white light interferometric (WLI) methods ${ }^{13-15}$ were developed that overcome the limitations of these PSI techniques. These interferometers allow surface profiling with high accuracy over a virtually unlimited range, making them more suitable for profiling stepped or discontinuous surfaces. One of the first techniques to utilize the short coherence of the white light source was the scanning interference microscope. ${ }^{16-18}$ This shares many features with confocal microscopy and has been used to inspect semiconductor wafers and integrated circuits. ${ }^{19}$ The method we use is based on another WLI profiler, called coherence radar. ${ }^{20}$ This detects the occurrence of interference while scanning the object in depth. It differs from conventional WLI in which depth accuracy is limited by the aperture. The entire image field is viewed at one time without the need for scanning the surface in both the $x$ and $y$ directions. However, a common problem is the vast amount of data, which greatly increases the acquisition and analysis time. Many different techniques have been used with these profilers to speed up the analysis, including Fourier and phase-shifting methods. Recently, a simple phase-shifting algorithm was developed that is highly effective and can be used with these WLI profilers. ${ }^{21}$
In this paper, we use two algorithms that do not involve Fourier or phase-shifting techniques. Instead, they deal with the data in a simple manner requiring very little analysis and involve simple computer programs, thus reducing the acquisition time. Note that we realize that white light profilers are commercially available. However, these profilers are usually limited to relatively smooth surfaces as well as being very expensive. They are normally scanning interferometers and do not image the whole surface at one time. The aim of this paper is to present a white light interferometric profiler that can be set up easily with standard optical equipment.

\section{Optical Setup}

The profiler is illustrated in Fig. 1. The optical setup is essentially a Michelson interferometer with one of the mirrors replaced by the surface to be profiled. The reference mirror is mounted on a computer controlled dc motor that enables it to be moved along the $z$ axis. A white light source with a coherence length of approximately $1 \mu \mathrm{m}$ is used. The rough surface is imaged onto a CCD camera and the image is then sent to the computer. Before starting a scan the helium-neon laser is used to ensure the reference mirror is aligned. The reference mirror is then moved to the end point of the scan and the laser is used to ensure it moves parallel to the $z$ axis. The reference mirror is then returned to the starting point. To achieve maximum contrast a neutral density filter is placed in the path to the reference mirror, so that the reflected intensities are approximately equal. However, this filter may distort the profile of the surface if it is not of high quality. One possible way of overcoming this is to use reference mirrors of appropriate reflectivity. The profiler uses the short coherence length of the light source. The visibility of the interference decreases rapidly from its maximum at 0 optical path difference to 0 at optical path differences greater than the coherence length.

In Fig. 1, if the light paths from point $P_{0}$ and from the reference mirror are equal, interference will be seen in the 


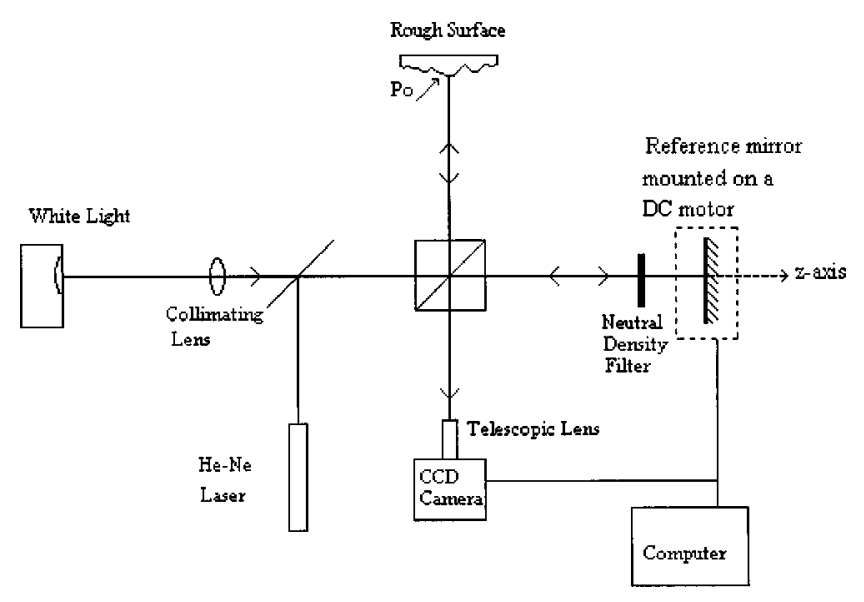

Fig. 1 Setup of the coherence radar.

pixels corresponding to that point and every other point on the surface for which the optical path difference is 0 . As the reference mirror is moved along the $z$ axis, the points of interference change to new points corresponding again to equal optical path lengths. Figure 2 shows a single pixel intensity graph obtained from a detector placed in the image plane of the profiler as the optical path difference is varied through 0 . The interference region occurs at 0 optical path difference and is only a couple of micrometers wide. For each pixel in the frame, this region must be identified and the corresponding frame position within the scan located. The rest of the distribution represents background illumination, and to speed up the acquisition and analysis times the profiler ignores this data. During profiling, the reference mirror is moved along the $z$ axis and frames are grabbed continuously. These frames are analyzed so that at the end of the scan, the interference region has been located for each pixel in terms of the frame number in which the interference, or coherence, region occurred.

\section{Acquisition and Analysis}

The first of the two algorithms locates the point of maximum modulation, within the coherence region, for each pixel in the frame. Figure 3 shows a typical interference signal. Four buffers, numbered 1 to 4 , are used as memory locations for data acquisition. Frames are continuously grabbed as the reference mirror is moved through a prede-

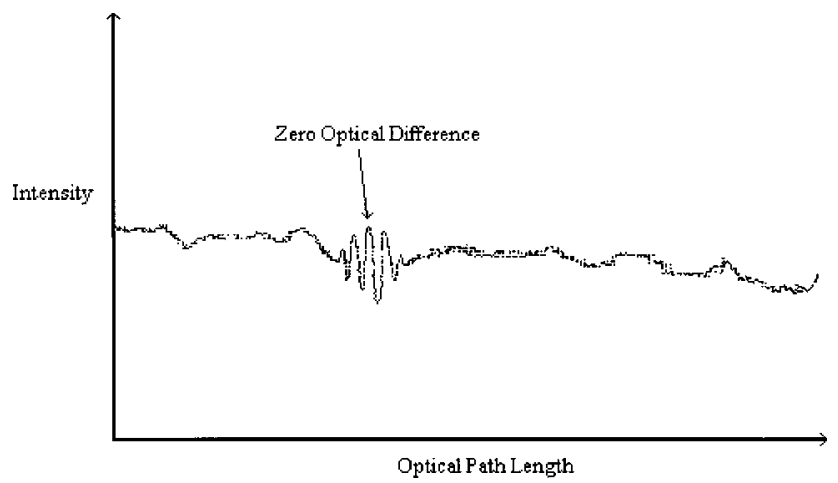

Fig. 2 Pixel intensity level as optical path length is varied through 0 optical path difference.

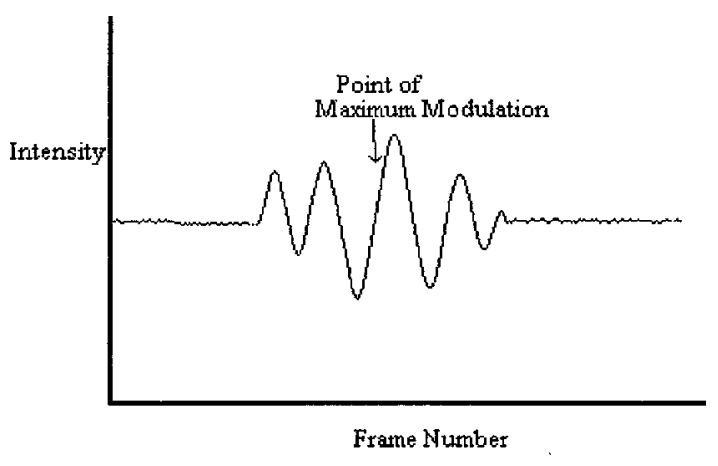

Fig. 3 Interference signal.

termined distance. For instance, if the scan length was 50 $\mu \mathrm{m}$, a frame could be grabbed every quarter of a micrometer. As each frame is grabbed, for every pixel in the frame, the intensity values are compared to the values that occurred in previous frames, so that at the end of the scan, for each pixel, the lowest and highest intensities and frame numbers in which these intensities occurred are stored in the four memory locations, buffers $1,2,3$, and 4, respectively. Buffers 3 and 4 are then compared to one another to ensure that, for every pixel, the difference, in terms of the frame number, between the location of the maximum intensity and the minimum intensity are a distance $\lambda / 4$ apart, where $\lambda$ is the mean wavelength of the source. Any pixel where the difference is greater than this value can be deleted and a neighboring pixel averaging technique can be used to fill in these pixels when the surface is imaged. The frames in which the peak intensities occurred for each pixel are then used to build an image of the surface. As the distance traveled by the reference mirror is precisely controlled, the relative positions of these intensities within the scan are known. A simple 3-D curve-fitting technique is used on these positions of maximum intensity to build an image of the surface.

The profiler builds an image of the surface using the points of peak intensity, which it detects with an accuracy of the step size taken between each frame. The profiler has a resolution that depends on the width of the peak intensity, providing the reference mirror can move in steps smaller than this width. Otherwise the resolution of the profiler is the minimum step size the reference mirror can move. Our profiler has a resolution of $0.06 \mu \mathrm{m}$, as this is the smallest step the reference mirror can move. This algorithm works well if the profiled surface is smooth, as in Fig. 4, and has been used successfully on smooth step heights.

If the surface is rough, however, scattering can cause peak intensities to occur outside the coherence region. On these surfaces we use an alternative algorithm that detects continuous modulation; that is, it detects the coherence region for every pixel in the scan. Again, it does this by continuously grabbing frames as the reference mirror is moved through a predetermined distance. As each frame is grabbed, the pixel intensity levels are recorded and compared to that of the previous frame. If the difference between the intensity levels is greater than a set threshold value, for any pixel, modulation is recorded. If the modulation is recorded for four consecutive frames, then the fourth frame number is sent to a memory location for that 


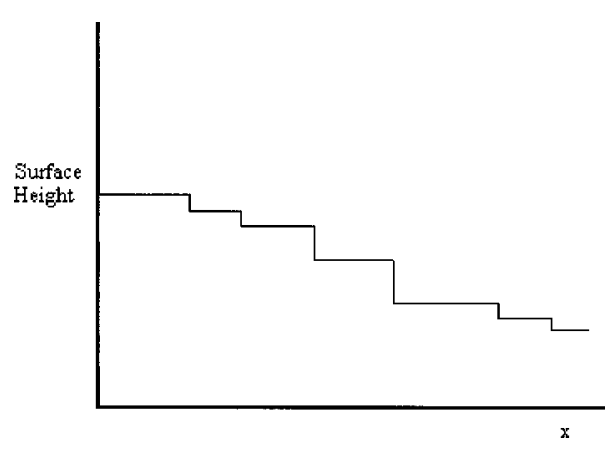

Fig. 4 This surface has a step profile but it is smooth.

pixel. As the distance traveled between each consecutive frame is controlled by the computer, the relative position of this frame is known. Then all these positions, one for each pixel, are used to build a 3-D image of the surface. The obvious disadvantage of this method compared to the previous algorithm is the poorer resolution. The profiler is now locating the coherence region that has a length of twice the coherence length of the source $l_{c}$. It does not know exactly where within this region the frame is, so there is an uncertainty in each reading of $\pm l_{c}$. This method has a resolution of an optical path change of $2 l_{c}$, which corresponds to a movement of the mirror of $l_{c}$.

When using the first algorithm, it is important that the distance that the reference mirror moves, between each frame, be small enough so that both the highest and lowest intensities are recorded. These intensities are a distance $\lambda / 2$ apart, which corresponds to a movement of the mirror of $\lambda / 4$, so this is the maximum step size. Using this step size, however, can result in a loss of relevant data. If the distance between the frames is very small, the data recorded gives an accurate description of the signal and so the results will be more accurate. In the second algorithm, the step size must be such that at least four frames are grabbed within the coherence region. It cannot be too small, however, or the modulation between consecutive frames will not be greater than the threshold value. These restrictions mean the acquisition time for the second algorithm is less than that for the first.

These algorithms work very quickly and use a minimum of memory as the frames are discarded immediately after they are analysed. When using the second program, if a high resolution is not required, the light source can be filtered or replaced by an IR source, thus increasing the coherence length and so reducing the number of frames needed for a complete scan, which in turn reduces the acquisition time. The first program can also be used with a bandpass filter, which lets through the desired frequency band only, and as the average wavelength is known, this band can be easily calculated. This enables the program to be used on rough surfaces, as it filters out all unwanted noise. The algorithms can be used in conjunction with one another so that the second algorithm detects the interference signal, and once this is located, the first algorithm locates the point of maximum modulation within this signal. This means the profiler now has a resolution of 0.06 $\mu \mathrm{m}$ even when profiling rough surfaces. Practical consider-

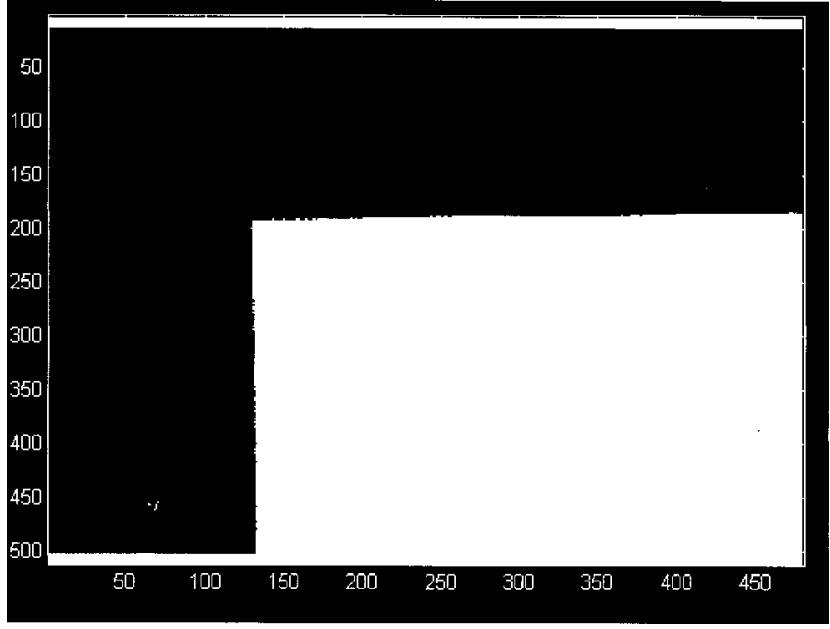

Fig. 5 Result from the first program of a scan of a step height. The gray level represents the frame number in which maximum modulation occurred. The $x$ and $y$ axes show pixel coordinates on the surface.

ations associated with coherence scanning are discussed by Dresel et al. ${ }^{20}$

\section{Results}

Figure 5 shows the results from the first program of a scan of a smooth step height. It shows the result on a 2-D scale where the pixel intensity level represents the frame number in which maximum modulation occurred. Figure 6 shows the same result in three dimensions. The height of the step, in terms of the frames, is 200. The distance traveled by the reference mirror between each frame was $0.5 \mu \mathrm{m}$, so the height of this step is $100 \mu \mathrm{m}$. Figure 7 shows the result of a scan of the letter $p$ from an Irish 10 pence piece. This represents a comparatively rough surface.

\section{Conclusion}

We developed two simple acquisition algorithms for WLI profilers. We have shown that they both work well with the coherence radar, simplifying the acquisition and setup. The profiler works with a resolution of $0.06 \mu \mathrm{m}$ and a range

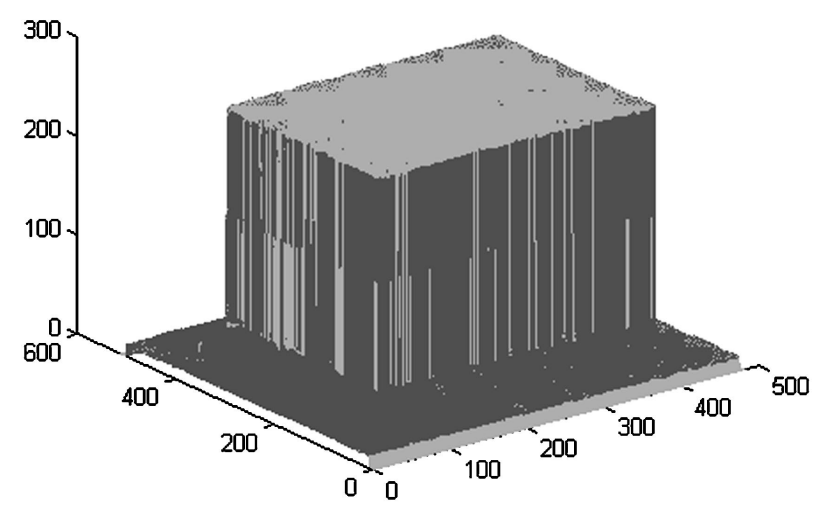

Fig. 6 Three-dimensional image of a surface with a step height of $100 \mu \mathrm{m}$. The $x$ and $y$ axes are shown in terms of the pixel positions and the $z$ axis represents the pixel intensity level. 


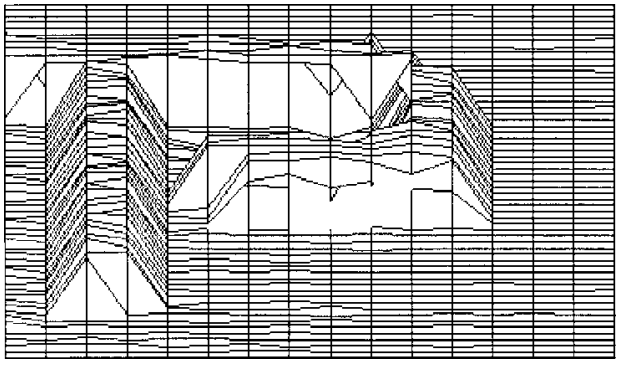

Fig. 7 Three-dimensional image of the letter $p$ taken from an Irish 10 pence coin.

limited only by how far the reference mirror can move, about $5 \mathrm{~cm}$ in our system. To scan over this distance it is advisable to use a high-quality dc motor moving at a relatively slow speed to ensure steady motion. A more advisable method is to use an Oriel "worm" controller, which is made up of a series of piezoelectric transducers (PZTs). The profiler does not have the problems associated with phase shifting and does not involve complex algorithms. Optical profilers can be very useful in any metrology laboratory. We have shown that the optical setup of a WLI profiler and the acquisition can be easy to setup and use. For optimum performance, the system should be made as small as possible with small distances between the beamsplitter and the reference mirror and surface. This reduces the errors caused by air turbulence and vibrations. The system should be well clamped and ideally should be constructed on an isolated optical table. The system will still work without fulfilling these conditions, but the errors will be noticeably greater.

\section{Acknowledgments}

We would like to thank Alfredo Lulli at the European Union Joint Research Centre, Ispra, Italy, for all his help. We would also like to acknowledge the support of the Dublin Institute of Technology's Strategic Research and Development programme.

\section{References}

1. H. Bruning, J. E. Gallagher, D. P. Rosenfeld, A. D. White, D. J Brangaccio, and D. R. Herriott, "Digital wavefront measuring interferometer for testing optical surfaces and lenses," Appl. Opt. 13, 2693-2703 (1974)

2. Y.-Y. Cheng and J. C. Wyant, "Phase shifter calibration in phaseshifting interferometry," Appl. Opt. 24, 3049-3052 (1985).

3. P. Hariharan, B. F. Oreb, and T. Eiju, "Digital phase-shifting interferometry: a simple error-compensating phase calculation algorithm," Appl. Opt. 26, 2504-2506 (1987).

4. J. Schweider, R. Burow, K. E. Elssner, J. Grzanna, R. Spolaczyk, and K. Merkel, "Digital wave-front measuring interferometry: some systematic error sources," Appl. Opt. 22, 3421-3432 (1983).
5. G. Häusler and J. Hutfless, M. Maul, and H. Weissmann, "Range sensing based on shearing interferometry," Appl. Opt. 27, 4638-4644 (1988).

6. A. F. Fercher, H. Z. Hu, and U. Vry, "Rough surface interferometry with a two-wavelength heterodyne speckle interferometer," Appl. Opt. 24, 2181-2188 (1985).

7. K. Creath, "Step height measurement using two-wavelength phaseshifting interferometry," Appl. Opt. 26, 2810-2816 (1987).

8. Y.-Y. Cheng and J. C. Wyant, "Multiple-wavelength phase shifting interferometry," Appl. Opt. 24, 804-806 (1985).

9. T. C. Strand and Y. Katzir, "Extended unambiguous range interferometry," Appl. Opt. 26, 4274-4281 (1987).

10. C. C. Williams and H. K. Wickramasinghe, "Optical ranging by wavelength multiplexed interferometry," J. Appl. Phys. 60, 19001902 (1986)

11. L. M. Frantz, A. A. Sawchuk, and W. von der Ohe, "Optical phase measurement in real time," Appl. Opt. 18, 3301-3306 (1979).

12. K. G. Larkin and B. F. Oreb, "Design and assessment of symmetrical phase-shifting algorithms," J. Opt. Soc. Am. A. 9, 1740-1748 (1992)

13. P. A. Flournoy, R. W. McClure, and G. Wyntes, "White-light interferometric thickness gauge," Appl. Opt. 9, 1907-1915 (1972).

14. B. L. Danielson and C. Y. Boisrobert, "Absolute optical ranging using low coherence interferometry,'” Appl. Opt. 30, 2975-2979 (1991).

15. L. M. Smith and C. C. Dobson, "Absolute displacement measurements using modulation of the spectrum of white light in a Michelson interferometer," Appl. Opt. 28, 3339-3342 (1989).

16. B. S. Lee and T. C. Strand, "Profilometry with a coherence scanning microscope," Appl. Opt. 29, 3784-3788 (1990).

17. S. S. C. Chim and G. S. Kino, "Three-dimensional image realization in interference microscopy," Appl. Opt. 31, 2550-2553 (1992).

18. G. S. Kino and S. S. C. Chim, "Mirau correlation microscope," Appl. Opt. 29, 3775-3783 (1990).

19. M. Davidson, K. Kaufman, L. Mazor, and F. Cohen, “An application of interference microscopy to integrated circuit inspection and metrology," in Integrated Circuit Metrology, Inspection and Process Control, Proc. SPIE 775, 223-247 (1987).

20. T. Dresel, G. Häusler, and H. Venzke, "Three dimensional sensing of rough surfaces by coherence radar,' Appl. Opt. 31, 919-925 (1992).

21. K. G. Larkin, "Efficient nonlinear algorithm for envelope detection in white light interferometry," J. Opt. Soc. Am. A. 13, 832-843 (1996).

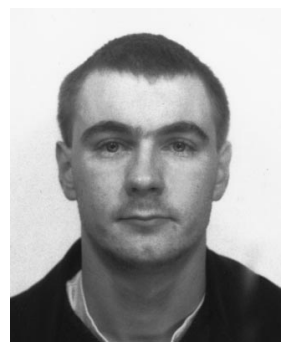

Brian Bowe received his BSc in physics and mathematics from Trinity College Dublin in 1995 and is now a PhD student working in the Laboratory for Holographic Applications in the Dublin Institute of Technology. His PhD research is in the area of optical metrology techniques. During his 2 years as a PhD student, he has spent 6 months working in the European Commission Joint Research Centre in Ispra, Italy.

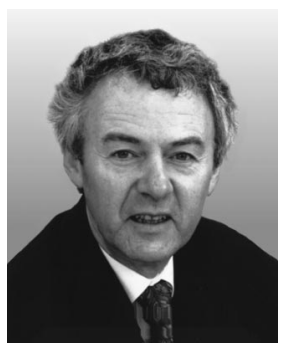

Vincent Toal received a BSc degree in experimental physics from the University College Dublin in 1968, an MSc in optoelectronics from Queen's University, Belfast, (in 1969), and a PhD in electronic engineering from the University of Surrey in 1976. He presently heads the School of Physics at the Dublin Institute of Technology and directs its Laboratory for Holographic Applications. The laboratory's main research interests are selfprocessing holographic recording materials and electronic speckle pattern interferometry and their applications. 